\title{
LA CONDICIÓN JUVENIL INDÍGENA: ELEMENTOS INICIALES PARA SU CONSTRUCCIÓN CONCEPTUAL
}

\author{
RENÉ UNDA LARA* \\ GERMÁN MUÑOZ
}

\begin{abstract}
RESUMEN
El presente artículo es producto de una investigación en curso en tres provincias de la Sierra Central del Ecuador en que se analizan tres procesos fundamentales que intervienen el espacio de las dinámicas comunales del mundo indígena andino: a) relaciones (neo) coloniales cifradas en el nombrar los sujetos y sus relaciones, en el instituir prácticas y en el administrar población; b) la relación con la tierra y sus distintas morfologías de reproducción socioeconómica y simbólica; c) la movilidad humana expresada en los procesos migratorios. Creemos que es en el marco de estos procesos articulados por formas modernizadoras, vehiculizadas desde la educación escolarizada y la mercantilización generalizada de las relaciones sociales, donde la constitución del sujeto joven-juvenil indígena podrá ser mejor comprendida.
\end{abstract}

PALABRAS CLAVE: COMUNIDAD, INDÍGENA, JÓVENES

* Sociólogo. Profesor investigador de la Universidad Politécnica Salesiana del Ecuador, Centro de Investigaciones de la Niñez, Adolescencia y Juventud (UPS), Ecuador. Director de la Maestría en Política Social de la Infancia y Adolescencia. Miembro del Grupo de Trabajo «Juventud y nuevas prácticas políticas en América Latina», CLACSO. Director de la investigación «Juventud indígena en el Ecuador», UPS/VME. Correo electrónico: reneunda78@gmail.com.

** Filósofo. Universidad San Buenaventura, Colombia. Doctor en Ciencias Sociales, Niñez y Juventud, CINDE/Universidad de Manizales. Profesor investigador de la Universidad Distrital Francisco José de Caldas, Bogotá. Profesor del Doctorado en Ciencias Sociales, Niñez y Juventud, CINDE/Universidad de Manizales y Coordinador del Grupo de Trabajo y de la Línea de Investigación «Jóvenes, culturas y poderes» del mismo doctorado. Correo electrónico: gmunozg2000@yahoo.es. 


\title{
A CONDIÇÃO JUVENIL INDÍGENA: ELEMENTOS INICIAIS PARA SUA CONSTRUÇÃO CONCEITUAL
}

\section{RESUMO}

Este artigo, resultado de uma pesquisa em andamento em três províncias da Sierra Central do Equador, analisa três processos fundamentais que atravessam o espaço das dinâmicas comunais do mundo indígena andino: a) relações (neo) coloniais codificadas no nomear os sujeitos e suas relações, no instituir práticas e no administrar a população; b) a relação com a terra e suas distintas morfologias de reprodução socioeconômica e simbólica; c) a mobilidade humana expressa nos processos migratórios. Nesses processos, articulados por formas modernizadoras veiculadas a partir da educação escolar e a mercantilização generalizada das relações sociais, é que acreditamos que a constituição do sujeito jovem-juvenil indígena poderá ser mais bem compreendida.

PALAVRAS CHAVE: COMUNIDADE, INDÍGENA, JOVENS

\section{THE CONDITION OF THE NATIVE YOUTH: INITIAL ELEMENTS FOR THEIR CONCEPTUAL CONSTRUCTION}

\begin{abstract}
This article is a product of investigations taking place in three provinces of the Central Sierra of Ecuador, analyzes three fundamental processes which through the space of communal dynamics of the native Andean world: a) relationships (neo) colonial figures in naming the subjects and their relations, instituting practices and in administration of the population; b) the relationship with the Earth and its distinct socio-economical and symbolical morphological reproduction; c) the human mobility expressed through migratory processes. In these processes, articulated by modernized forms from school education and general mercantile c). In these processes, articulated by modernized forms of transportation, from school education and the generalized mercantile of social relations that constitutes a young native, we believe, can be better understood.
\end{abstract}

KEY WORDS: COMMUNITY, NATIVE, YOUTH 


\section{INTRODUCCIÓN}

¿QUÉ PROCESOS DEFINEN LA categoría joven en el contexto de las comunidades indígenas de la Sierra Central ecuatoriana? ¿Qué es ser joven y qué es la juventud para los habitantes de las comunidades indígenas de la Sierra Central ecuatoriana? ¿Qué prácticas socioculturales desarrollan los/as jóvenes adolescentes indígenas en los espacios en los que desarrollan sus experiencias vitales?

A partir de estas interrogantes, la investigación en la que se inscriben los resultados parciales presentados en este artículo pretende identificar y analizar el entramado de elementos socioculturales que constituyen la categoría joven en comunidades indígenas en un escenario general de transformaciones de estas comunidades, producto de las actuales dinámicas locales que configuran nuevas fronteras territoriales y simbólicas entre lo rural y lo urbano. En el caso de las comunidades indígenas que se estudian en este trabajo, la cuestión de la construcción de la juventud y de lo joven se encuentra atravesada por problemáticas de alcance global como la migración, la precarización del trabajo y del mercado laboral, así como por fuertes tensiones identitarias resultantes de las nuevas relaciones entre lo urbano-rural y de los sujetos que las dinamizan.

Las primeras aproximaciones al contexto de estudio permiten advertir la presencia de formas y prácticas socioculturales mixturizadas desde matrices rurales y urbanas. En tales dinámicas, suponemos, se constituye el sujeto joven de las comunidades indígenas con sus propias especificidades respecto de las nociones mediante las cuales se han impuesto las representaciones y discursos hegemónicos sobre los/as jóvenes en el campo de estudios de juventud.

La aludida mixturización del mundo indígena puede observarse y ser analizada únicamente en clave comparativa bajo una doble estrategia diacrónica y sincrónica. La mirada diacrónica permite apreciar los cambios que se han producido en la comunidad andina indígena a lo largo de las últimas décadas, y la mirada sincrónica posibilita situar las múltiples intersecciones de lo rural y de lo urbano, de lo indígena y de lo mestizo, de lo campesino y de lo citadino en un momento particular de la historia.

Este ejercicio permite ir dando forma a un dispositivo conceptual, teórico y metodológico capaz de activar comprensiones acerca de la evolución del proceso de transformaciones de la comunidad indígena y propiciar explicaciones sobre las actuales prácticas socioculturales de 
los/as jóvenes indígenas desde un enfoque investigativo predominantemente inductivo. Se trata, en rigor, de un dispositivo epistemológico con el que podamos dar cuenta de las prácticas sociales de la juventud indígena como parte de los cambios y transformaciones de la comunidad indígena. A la vez, tales cambios constituyen el marco de comprensiones posibles acerca de las prácticas socioculturales de los jóvenes.

La investigación en curso toma en cuenta los principales cambios y transformaciones producidos en la comunidad andina a partir de estudios, análisis e investigaciones realizados en Ecuador desde inicios de la década de 1980, cuyos aportes están sirviendo como guías orientadoras en los planos conceptual, metodológico y procedimental para reforzar o contrastar sus desarrollos. Puesto que este artículo, a partir de las consideraciones conceptuales expuestas, presenta un primer avance de la mencionada investigación, nos interesa mostrar una breve caracterización del espacio social de lo indígena a través de tres cuestiones que consideramos han estado presentes de modo más o menos constante - aunque con diversos matices - en estudios precedentes sobre la comunidad andina (Murra, 1975; Oberem, 1979; Martínez, 1980; Plaza, 1981; Salomon, 1981; Sánchez Parga, 1984, 1993, 2002, 2007) y que constituyen los ejes analíticos desde los que intentamos comprender las prácticas socioculturales de jóvenes indígenas en tres provincias de la Sierra Central ecuatoriana, a saber: la actualidad de una matriz colonial que recubre las relaciones entre jóvenes y sociedad comunal; el carácter actual de los jóvenes indígenas y su relación con la tierra, es decir, con el elemento central de la relación comunal y comunitaria; y desde el campo de los flujos migratorios en el que las prácticas y acciones de los jóvenes indígenas van adquiriendo cada vez con mayores intensidades características inscritas en prácticas de riesgo (Beck, 2002).

\section{ESPACIO SOCIAL DE LO INDÍGENA EN EL CONTEXTO DE LA INVESTIGACIÓN}

La categoría de espacio social acuñada por Milton Santos (1996) permite ubicarnos en los múltiples puntos de intersección entre los planos de lo geográfico, lo histórico, lo cultural y lo sociopolítico en una determinada temporalidad. Una caracterización inicial de las comunidades indígenas en las que estamos desarrollando la investigación en la que se enmarca este trabajo, toma en cuenta el espacio social como categoría útil para comprender el conjunto de condiciones generales 
en las que viven actualmente dichas comunidades desde las distintas perspectivas ya mencionadas.

Tal categoría permite desarrollar, a la vez, vías analíticas y explicativas acerca de la cuestión indígena con relación al contexto más amplio del Estado ecuatoriano. El espacio social es un espacio siempre dinámico, producto y productor de cambios y transformaciones sociales en sus distintos ámbitos y escalas. La cuestión indígena en el Ecuador sólo puede ser comprendida en su relación con las estructuras, prácticas y discursos producidos en el marco de la etapa colonial y republicana. Nuestro estudio considera el análisis de los principales procesos ocurridos desde la década de 1980 hasta el momento de la realización de la investigación (julio-diciembre 2010), aunque en varios tramos debamos referirnos a eventos y acontecimientos anteriores al corte temporal previsto en este estudio.

La investigación se desarrolla en comunidades indígenas (104) de tres provincias de la Sierra Central del Ecuador (Cotopaxi, Tungurahua y Chimborazo). Son provincias en las que por razones de índole socio-histórico y cultural mejor se expresa la historia de la comunidad andina indígena. Aunque «ninguna comunidad es igual a otra», ${ }^{1}$ todas comparten, de modos distintos, las mismas matrices histórico culturales en las que se han desarrollado ciertas instituciones del coloniaje como el concertaje y la encomienda y posteriormente la hacienda (Albornoz, 1971).

Es en la relación con la tierra, en el conjunto general de sus actividades inscriptas en la reproducción material y simbólica de la sociedad comunal, que se reconoce una de las dimensiones identitarias más profundas de lo indígena. La comunidad indígena, en tanto espacio social, se encuentra atravesada por prácticas de producción y reproducción social vinculadas con los usos de la tierra.

Cuando el uso productivo, de subsistencia o económico del suelo es mínimo, o se ha precarizado a niveles que no permiten la reproducción de las condiciones de vida básicas de la familia o de la comunidad, se produce — con intensidades variables según familias y comunidades- la migración hacia los centros parroquiales, a las cabeceras cantonales, a las capitales de provincia o hacia el extranjero.

1 Esta importante apreciación empírica, corroborada en algunos estudios como los de Luciano Martínez (1992) o de José Sánchez Parga (2002) se la debo a René Unda Proaño, quien acredita un trabajo de cerca de cuatro décadas con comunidades indígenas y campesinas del Ecuador. 
No es una tarea sencilla advertir en los flujos migratorios de la comunidad andina indígena patrones regulares y uniformes de movilidad desde los que puedan establecerse generalizaciones rígidas. El espectro de posibilidades y variantes es amplio y en no pocos casos cambiante en marcos temporales relativamente cortos. Entre las comunidades en las que existe una larga y sostenida experiencia migratoria hacia los centros urbanos del país o del extranjero y aquéllas en las que los flujos migratorios han constituido un fenómeno de baja intensidad, existen múltiples situaciones intermedias en una misma provincia, en un mismo cantón y parroquia, e incluso, entre comunidades vecinas. En cualquier caso, el fenómeno migratorio representa uno de los principales problemas para la comunidad indígena por las características que este tipo de movilidad forzada comporta.

Tenemos entonces que a partir de la categoría de espacio social, entendida como un cronotopos que desborda lo estrictamente geográfico, podemos ir identificando algunos de los ejes de caracterización de las comunidades con las que desarrollamos la investigación: i) la comunidad indígena andina como elemento constitutivo de una matriz de relación social de coloniaje que se prolonga, bajo distintas morfologías, en el período republicano temprano y reciente; ii) la comunidad indígena y su compleja relación con la tierra, cuestión que, claramente, va más allá del «uso del suelo»; iii) comunidades humanas con capacidades tácticas o estratégicas de movilidad territorial.

En torno de estos tres ejes de caracterización se organiza todo un sistema de disposiciones conformado por campos y agentes cuyas prácticas y acciones han ido cambiando o modificando los distintos campos constitutivos de la comunidad andina indígena, siempre en relación con campos y fuerzas de otros espacios sociales. Se trata, por supuesto, de una caracterización estructural cuya función es servir como ejes analíticos de orden investigativo y explicativo.

\section{MATRIZ COLONIAL DE LA COMUNIDAD ANDINA INDÍGENA}

\section{a) Nombrar}

La misma forma de nombrar a los habitantes nativos - «indios», luego «indígenas»— en lo que hoy conocemos como América Latina, plantea ya uno de los problemas centrales del coloniaje porque en aquello que se nombra opera siempre una relación intersubjetiva de poder (Foucault, 1986; Wodak, 2005). Los órdenes discursivos supo- 
nen la realización de actos de enunciación y de actos de habla cuya función es configurar contratos sociales de comunicación y de habla mediante los cuales se produce un reconocimiento intersubjetivo, campos de sentido y, en definitiva, lenguaje compartido entre sujetos.

Los indígenas fueron nombrados así por una urgente y casi automática necesidad de administrar la población por parte de las distintas instituciones de la colonia y de la república. En ese proceso se desconoce, se niega y se ignora al otro cultural que ha sabido producir sus propias denominaciones. Y también se producen integraciones asimétricas, problemáticas.

El reconocimiento jurídico de pueblos y nacionalidades, plasmado en la Constitución y leyes del Ecuador desde 1998, supuso la activación de una progresiva visibilización de las particularidades y especificidad de los diversos pueblos y nacionalidades indígenas del Ecuador, así como de su pluralidad interna constitutiva. No obstante, la relación con las estructuras y dinámicas del Estado Nacional y con otros espacios institucionales, tales como las agencias de cooperación internacional y los medios masivos de comunicación, ha impuesto que la denominación genérica de indígenas se mantenga.

En el orden de la representación política, tanto en la dinámica de movimiento social (Confederación de Nacionalidades Indígenas del Ecuador) como en la del movimiento-partido político (Pachakutik), la necesidad de nombrarse indígena ha sido una cuestión en la que convergen procesos identificatorios desde abajo, en su condición de sujetos subalternizados y perspectivas pragmáticas de articulación con el orden y las prácticas políticas del sistema político ecuatoriano.

\section{b) Instituir}

Las instituciones de la colonia, entre ellas el concertaje y la encomienda, constituyeron los espacios de concreción en los que lo indígena, en tanto forma cultural, y los indígenas, como sujetos empíricos, ocuparon lugares determinados en la sociedad colonial. Tales lugares eran, de modo casi absoluto, el sometimiento y la explotación como los modos privilegiados de relación social y fuente de interculturalidades. Nos interesa subrayar la importancia de estos dos tipos de institución colonial —el concertaje y la encomienda- puesto que constituyen los antecedentes más directamente vinculados con la hacienda y con los más recientes procesos de redistribuciones y lucha por la tierra. 
En el entramado de las prácticas por la ocupación, posesión y usos de la tierra se encuentra presente, como en toda producción social, la dimensión simbólica (afectos, vínculos, memoria) de relación con la tierra, cuestión que, provisionalmente, permite conjeturar acerca de por qué muchas comunidades indígenas —a pesar de las duras condiciones de vida de sus habitantes- no desaparecen ni dan muestras de desaparecer en un futuro próximo.

\section{c) Administrar}

Con el advenimiento de la República, las condiciones de vida de la población indígena se mantuvieron signadas por la dominación y el sometimiento por parte de los estamentos de criollos, quienes pasaron a ejercer la conducción política y económica del naciente Estado. La alternancia en el ejercicio de gobierno entre las élites dominantes de la Sierra y de la Costa no sigue patrones definidos de regularidad, pero marca una tendencia que tiene sus cortes en los períodos de los gobiernos militares. Entre 1830 y 1900, las condiciones de vida de la mayoría de la población indígena se precarizan en medio de las permanentes redistribuciones territoriales de los enclaves productivos y comerciales. La población indígena o bien forma parte de la estructura hacendataria en calidad de trabajadora servil, o se encuentra segmentada en proporciones menores en diversos oficios en los que se requiere fuerza y resistencia física.

La Revolución Liberal de Alfaro, cuyo punto culminante fue el 5 de junio de 1895, no fue suficiente para instaurar un proyecto político cultural que permitiese el reconocimiento de los indígenas como ciudadanos de derecho pleno, pero quedó la huella histórica en la memoria colectiva acerca de la posibilidad de destruir-construir nuevos órdenes sociales y políticos.

Acontecimientos como la Ley de Organizaciones y Comunidades Indígenas aprobada en 1937, los asentamientos y el poblamiento de zonas geográficas ubicadas entre el callejón interandino y el litoral, como parte de los primeros flujos migratorios importantes de las comunidades de zona alta hacia la Costa, entre otros, informan sobre una cada vez más evidente capacidad proactiva de articulaciones de importantes sectores indígenas, especialmente de la Sierra, con las dinámicas del Estado nacional.

El proceso de Reforma Agraria, entre los años sesenta y setenta del siglo pasado, a la vez que aceleró el desmantelamiento de la es- 
tructura hacendataria clásica, basada en el latifundio y en formas de concertaje de la redistribución —asimétrica — de los productos agrícolas, generó fuentes de mayor precarización para la vida de las comunidades indígenas al quedar liberados para vender su fuerza de trabajo en ausencia de condiciones laborales que garanticen la reproducción económica de la familia (Martínez, 1984). La creciente presencia de indígenas en los centros urbanos, especialmente en Quito y Guayaquil, debido, sobre todo, a la inexistencia de condiciones básicas para hacer producir sus minifundios y a la oferta de mano de obra barata en el sector de la construcción en el contexto general de la modernización socioeconómica del país, puso en evidencia un nuevo ciclo de transformaciones de la comunidad andina indígena.

A los flujos migratorios comunidad andina-centros urbanos debe sumarse la presencia de grupos religiosos evangélicos, mormones, adventistas, pentecostales, etcétera, de procedencia norteamericana, que desde los inicios de los años setenta promueven procesos de catequización que si bien, en principio, generan división en las comunidades indígenas, en el balance general actual que hacen las mismas comunidades parecería que los resultados son positivos, sobre todo por su incidencia en la disminución del consumo de alcohol.

En los años ochenta y noventa del siglo XX, el protagonismo del movimiento indígena es indiscutible en el Ecuador. Durante la década de 1980, el movimiento indígena moviliza sus acciones en clave de reivindicación identitaria (Sánchez Parga, 2007), cuestión que, en modo alguno, significa que dichas acciones carezcan de efectos políticos. Muy al contrario de lo que podría pensarse, el conjunto de demandas de corte identitario, de presencia de lo indígena en la vida nacional, constituyen la base y la entrada estratégica para que en la década de 1990 la institucionalización política del movimiento indígena definiera su programa a través de la presencia del Movimiento Político Pachakutik.

Desde entonces, la Confederación de Organizaciones y Nacionalidades Indígenas del Ecuador (CONAIE) y su brazo político (Pachakutik), sitúan a la plurinacionalidad como eje de sus reivindicaciones y demandas políticas. El reconocimiento constitucional de la especificidad cultural de los pueblos indígenas viene aparejado de una serie de respaldos legales derivados de la lucha por el reconocimiento de sus derechos colectivos.

La Constitución de 1998 y la actual, aprobada mediante plebiscito el 28 de septiembre de 2008, reconoce jurídicamente el hecho plurinacional en el marco de un estado unitario. 
El problema radica en la traducción política, territorial y administrativa de lo plurinacional, sobre todo en lo que dice relación con la explotación de hidrocarburos y minerales, procesos en los que los pueblos y nacionalidades indígenas se ven especialmente afectados. De igual forma, el reordenamiento territorial previsto en la Constitución del Sumak Kausay, o Buen Vivir, supone la activación de un complejísimo proceso en el que se cruzan las dimensiones geográfico-territorialeconómica, y por supuesto lo político-cultural. Lo plurinacional constituye, actualmente, un campo de disputa en el que las fuerzas interactuantes parecen no definir aún con la suficiente claridad los alcances y contornos del proceso de reconfiguración del Estado.

Es en esta conflictiva encrucijada de fuerzas y procesos donde intentamos comprender qué es ser joven hoy y cómo se construye la categoría juventud en la comunidad indígena andina de la Sierra Central ecuatoriana. Esta perspectiva socio-histórica y política permitirá plantear líneas explicativas sobre las relaciones entre escolarización, migraciones, consumos y configuraciones identitarias de las juventudes indígenas o, de modo más preciso, de la condición juvenil indígena en la actualidad.

\section{LA COMPLEJA RELACIÓN CON LA TIERRA}

Transcurridos casi dos meses del trabajo etnográfico sobre el terreno, la aparente inocencia de la antigua pregunta acerca de por qué la gente de las comunidades vive en lugares tan remotos y en medio de tan difíciles condiciones de reproducción social comunitaria, las antiguas respuestas aparecen válidas y siempre actuales:

Lo único que tenemos es nuestra parcela. Más antes, en tiempos de mis papás y mis abuelos, no teníamos ni eso. Ellos trabajaban para el patrón toda la vida y no les pagaban nada. En el año de 1973 empezaron a dar tierras y en 1982 ya soltaron a todos de la hacienda. Unas tierras compramos, otras nos entregó el IERAC. No tenemos agüita, hay que acarrear para cocinar, tomar. Sólo vivimos esperando el agua del cielo porque las gestiones con instituciones han quedado ahí o no terminan todavía. Entonces toca salir, ir a trabajar en Latacunga o Quito, siempre estamos dando la vuelta.

(Entrevista a dirigente indígena del sector de Guayama San Pedro, comunidad perteneciente a la parroquia Chugchilán, Cantón Sigchos, Provincia de Cotopaxi). 
Sin duda, la tierra ha sido el elemento central de relación social del mundo indígena. La historia de la comunidad andina indígena está indisociablemente ligada a su relación con la tierra por una multiplicidad de razones en las que se intersectan las estrategias de reproducción económica, en su condición de propiedad de la hacienda, ${ }^{2}$ de huasipungueros ${ }^{3}$ o de indios libres, ${ }^{4}$ su reproducción identitaria, cultural y simbólica cada vez más permeada por relaciones con la ciudad y con lo urbano, activadas sobre todo por los flujos migratorios.

Como estrategia de reproducción económica, la relación con la tierra está sujeta a un campo de variabilidades que expresan las distintas condiciones entre las comunidades indígenas en un momento dado y sus cambios a lo largo del tiempo. En el caso de las comunidades de las tres provincias de la Sierra Central ecuatoriana en las que estamos desarrollando la investigación, se evidencia que una de las características de la relación con la tierra es su condición de creciente precariedad como tendencia dominante.

El principal y más generalizado problema de tales comunidades, en las que la tradición hacendataria tuvo una marcada presencia y repercusión, es la escasa disponibilidad de agua y el difícil acceso a las fuentes de este recurso fundamental. Antes que los problemas que los jóvenes actuales pueden representar para las comunidades está el problema del agua, y es a partir de su agudización que la relación con la tierra adquiere nuevas características debido a un progresivo distanciamiento, especialmente de los varones, al tener que salir de su comunidad en busca de recursos que permitan la subsistencia de sus familias.

2 Entendido esto como «objeto de propiedad» del patrón o dueño de la forma y estructura de propiedad llamada hacienda.

3 El huasipungo («casa pequeña-propia») constituye una referencia de primera importancia en la relación del indígena con la tierra, porque más allá de su característica precariedad en cuanto a carencias y ausencias de los factores necesarios para convertirlo en «unidad productiva», es el espacio físico y simbólico en el que el indio se reconoce como parte de una estructura hacendataria o como «indio libre» durante y luego del proceso de Reforma Agraria.

4 «Indios libres»: denominación que ha sido utilizada en algunos estudios como los de Udo Oberem (1979) y J. Sánchez Parga (2002) en sus investigaciones en la provincia de Cotopaxi y cuyo ambiguo significado está asociado, por una parte, a su emancipación respecto de la estructura hacendataria y, por otra, a una suerte de condena e imposibilidad: ser «indio libre» supone dejar de pertenecer a la comunidad. 
Las construcciones discursivas acerca de lo joven en estas comunidades se muestran muy asociadas a los cambios y transformaciones experimentados en relación con las difíciles condiciones de reproducción material de sus habitantes. Los jóvenes, desde hace dos o tres décadas, dependiendo de la comunidad a la que pertenezcan, empiezan a ser vistos, cada vez más, como sujetos en tránsito por los adultos actuales, quienes, a su vez, también han vivido la experiencia migratoria.

[...] de guambras íbamos de cargadores a Latacunga y Quito, de estibadores tan íbamos a Guayaquil. Ahora los jóvenes se van a La Maná, a la fábrica o al molino, otros van más lejos y algunos van a estudiar el colegio. Sí teníamos más antes agüita porque llovía y podíamos sembrar. Después entró ya la sequía, se hizo más duro y tocó salir pero siempre estamos dando la vuelta, no nos quedamos mucho nosotros.

(Comunero de Chaupi, parroquia Chugchilán, Cantón Sigchos, provincia de Cotopaxi).

En este fragmento de relato pueden apreciarse algunos elementos de gran interés para los objetivos de nuestro trabajo. Por un lado, la experiencia común de haber emigrado y, al mismo tiempo, el carácter distintivo de esta experiencia en términos generacionales. La migración de antes no es la misma que la de ahora y, por ello, los jóvenes estarían siendo distintos de lo que los adultos fueron cuando jóvenes. Y, evidentemente, distintos de los adultos actuales.

Estas prácticas — casi forzadas- de distinción tienen en su base un conjunto de motivaciones ligadas a la reproducción material de las familias y de la comunidad. No obstante, lo que para los adultos aparece como el principal problema de los jóvenes actuales es la «pérdida de la identidad cultural».

(Profesor indígena del Colegio Intercultural Bilingüe Jatari Unancha).

No existe narración o relato, por breve que sea, en la que los adultos no enuncien que el principal problema de los jóvenes de sus comunidades es que ya no usan sombrero ni poncho y que ahora todos andan zapatados; es decir, han dejado de usar alpargatas o, simplemente, usan zapatos. Esto lo señalan en tono casi acusatorio aún cuando ellos - los adultos - también han dejado de usar poncho, sombrero, y usen calzado similar al de los jóvenes.

A medida que prosigue la conversación y se ha acumulado un cierto capital de confianza dialogal, los adultos reconocen que ellos 
también han dejado de usar su indumentaria tradicional, al tiempo que van enunciando una serie de justificaciones que conducen a reconocer que la comunidad en su conjunto es la que se ha transformado.

Sin embargo, aquello no les impide reiterar el hecho de que son los jóvenes quienes han introducido los cambios más notables o drásticos en las comunidades y, consecuentemente, marcar sus diferencias con las jóvenes generaciones.

El conjunto de valoraciones que los adultos construyen en torno de las jóvenes de sus comunidades es aparentemente ambiguo, por lo menos en un primer momento. La ropa, la música, el uso de objetos como el celular, el habla, las aspiraciones, constituyen factores disruptivos en la relación intergeneracional que expresan tensiones de orden cultural al interior de la comunidad indígena y que, habitualmente, son observados y narrados por los adultos como una amenaza, como algo que debe ser amonestado por la comunidad y que merece revisarse.

[...] cuando vuelven ya vienen con cosas de la ciudad, aprenden otras costumbres. Algunos vienen poniendo arete. Unos jóvenes se habían puesto aretes entonces llamamos a los papás, a los compañeros y a ellos mismos les hicimos ver que no deben andar poniéndose eso. Sólo uno no ha hecho caso pero poco viene por acá.

(Dirigente adulto de comunidad Pilapuchin, parroquia Chugchilán, cantón Sigchos, Provincia de Cotopaxi).

El contrapeso de este conflicto cultural está representado por el conjunto de valoraciones que sitúan la educación como la cuestión de principal importancia en la vida familiar y comunal. Es decir, la tenaz lucha por dar educación a los hijos, especialmente secundaria y en muy pocos casos universitaria, encuentra en su trayecto una serie de elementos que ponen en riesgo un orden cultural comunal.

Puesto que el proceso educativo escolarizado se da normalmente fuera de las comunidades, porque el Estado no alcanza a cubrir las demandas y necesidades educativas del nivel medio, los jóvenes permanecen la mayor parte de su tiempo en los centros parroquiales, en las cabeceras cantonales o directamente en las ciudades capitales de provincia. Allí se encuentran expuestos a todo lo que el mercado les ofrezca bajo sus distintas formas y mediaciones. El consumo de objetos se convierte en una de las principales estrategias de integración frente a un paisaje sociocultural que las más de las veces se muestra hostil y agresivo. 
El carácter de dicha integración no es precisamente integrativo sino defensivo. Se trata, por un lado, de construir cercos defensivos frente a la marginación, exclusiones y discriminación de la que generalmente han sido objeto desde tempranas edades y, por otro, de explorar la novedad representada en la oferta de objetos de consumo.

Bajo las condiciones descritas, la relación de los jóvenes con la tierra, que es parte de la relación con la comunidad y con su familia, ha reforzado un carácter predominantemente simbólico en el que la dimensión socioeconómica ha sido relegada a un segundo plano. En términos de prácticas socioculturales, esto significa que campos como las festividades, las conmemoraciones, los matrimonios y bautizos —en los que, por cierto, está presente un determinado capital económico- son aquéllos en los que se advierte mayor presencia de los jóvenes.

Sin embargo, mayor presencia de los jóvenes en eventos como los señalados no significa necesariamente mayor participación en el conjunto de relaciones de vinculación simbólica (autoridad, compadrazgo, padrinazgo, priostazgo) ni en las relaciones de carácter económico-monetario (contribuciones materiales para la reproducción de dichos campos).

Es quizá por esta razón que en tales campos se evidencie con mayor fuerza el peso de la tradición. Con Bourdieu (1998) podríamos afirmar que el peso de los habitus estructurantes es relativa y sensiblemente menor que el de los habitus estructurados aunque debamos reconocer que en cada uno de estos campos (p. e. fiestas patronales, celebraciones matrimoniales) la relación entre habitus estructurados y habitus estructurantes sea distinta.

Existe, pues, un tejido comunitario de geometrías variables en torno de las prácticas que se configuran sobre la base de habitus instituyentes y de las prácticas instituidas. En tales relaciones, creemos, es donde se encuentran instaladas varias de las principales problemáticas desde las que se procesa y redefine la comunidad andina indígena en cuanto a su carácter y sentido comunal, y en cuanto al grado de (des) integración con referencia a procesos de modernización desruralizante y descampesinizador bajo una matriz de relaciones sociales marcadamente urbana.

Con referencia a la dimensión socioeconómica y sus actividades correspondientes, la relación de los jóvenes con la tierra está estrechamente vinculada con el generalizado proceso de precarización del agro (Martínez, 1984), cuyo núcleo más sensible es el minifundio y la pequeña propiedad de la tierra. 
En el mejor de los casos, la propiedad parcelaria, o de pequeños solares, sirve para atenuar las carencias relativas a la alimentación básica y, muy eventualmente, para sacar ciertos productos al mercado. El problema fundamental, como se ha indicado ya, es la falta de agua, sea en términos de difícil acceso a las fuentes o en términos de una inadecuada distribución debido, sobre todo, a la ausencia de la infraestructura necesaria para tales efectos.

En estas condiciones, los jóvenes representan trabajo productivo cesante e, incluso, trabajo social necesario no utilizable, cuestión que determina la migración hacia centros urbanos donde, bajo condiciones de precariedad laboral, se incorporan a trabajos relacionados con la construcción y trabajos de carga. El regreso periódico y temporal a sus comunidades se produce, entonces, con ocasión de las festividades y de eventos en los que la dimensión de vinculación simbólica es primordial.

\section{PARTIR, VOLVER, PARTIR...}

La comprensión y explicación de los flujos migratorios de las comunidades indígenas supone, por una parte, situar este fenómeno en la dinámica general de los procesos de movilidad humana de la sociedad moderna (Sassen, 2007) y, por otra, la puesta en acto de una actitud etnográfica que permita conocer desde adentro el conjunto de motivos, tácticas y estrategias que constituyen las acciones y decisiones de los sujetos que migran.

Aunque el fenómeno migratorio rural-urbano e, incluso, transnacional, no es nuevo en el Ecuador, es en la década de 1970, con el proceso de modernización socioeconómica, que los flujos migratorios internos adquieren regularidades que, posteriormente, irán modificándose tanto por la intensificación del fenómeno cuanto por el ensanchamiento de los rangos etarios y la creciente presencia de mujeres que migran.

En la base del proceso modernizador se encuentran las transformaciones estructurales de orden económico producidas por los cambios en el esquema general de intercambio comercial internacional que supuso el paso de una economía primaria de agroexportación hacia una economía primaria fundamentalmente extractiva, basada en la explotación de petróleo (Acosta, 1987).

La convergencia conflictiva de varios factores como una reforma agraria que liberó a los indígenas de las estructuras hacendatarias para colocarlos como mano de obra y fuerza de trabajo potencial y, sobre todo, vulnerable en el mercado de trabajo — puesto que las reducidas 
extensiones de terreno que les fueron asignadas no eran suficientes para la susbistencia familiar-, así como una tan inédita como amplia demanda de trabajadores para el sector de la construcción, configuró un nuevo ciclo en los procesos migratorios internos en el caso ecuatoriano.

El acelerado proceso de urbanización experimentado durante los años setenta en las dos ciudades más grandes del Ecuador — Quito y Guayaquil— supuso la absorción de población rural, en particular de población de las comunidades andinas indígenas de la sierra central y austral que, tras el proceso de reforma agraria y la generalizada fragmentación de la tierra en minifundios, se vio forzada a incorporarse a la construcción de urbanizaciones, ciudadelas y edificios en calidad de albañiles y peones, así como también a tareas de estibadores y cargadores en el puerto de Guayaquil y en diferentes mercados y plazas de las ciudades.

Durante las décadas de 1970 y de 1980 existen poderosas razones y abundantes evidencias que apoyan la tesis según la cual una abrupta descampesinización de la comunidad indígena se corresponde con una precaria proletarización de dicha población (Martínez, 1980). Aunque esta tesis ha sido objeto de cuestionamientos debido, sobre todo, al hecho constatable de ausencias relativas de condiciones de proletarización, cabe reconocer que, desde una mirada sociológica y antropológica, el desplazamiento de una descampesinización hacia una proletarización resulta, por decir lo menos, en una figura conceptual audaz y sugerente.

Desde mediados de la década de 1990 empieza a producirse un ensanchamiento de la franja etaria migrante y de la composición de la población migrante según sexo. Si en los años precedentes los varones a partir de los quince a dieciocho años salían de las comunidades, desde el período señalado los varones emigran desde los diez a doce años, y aún antes en ciertas comunidades, y las mujeres que emigraban eventualmente salen en proporciones similares a los varones (Sánchez Parga, 2002).

Las regularidades del hecho migratorio en esta dinámica particular que articula conflictivamente lo rural, lo campesino y lo indígena con lo urbano, lo (sub) proletarizado y lo indígena mixturizado, reivindicado o negado, están enmarcadas en la constante necesidad de salir de la comunidad, regresar cuando sea posible y volver a salir para subsistir.

En este ciclo se presentan múltiples situaciones que caracterizan el fenómeno migratorio de cada comunidad: migraciones de ciclo corto o largo, migraciones de ambos sexos, migraciones de rangos 
etarios diferenciados, migraciones según demanda de trabajo localizada, migraciones unipersonales o familiares. Invariablemente, cada tipo de flujo migratorio está determinado por el conjunto de condiciones particulares existentes en cada comunidad.

Los jóvenes indígenas, como producto histórico generacional concretamente situado, se encuentran sujetos a las determinaciones estructurales que afectan al conjunto de la población ubicada en los quintiles con menores ingresos monetarios. Con menores posesiones materiales más o menos fijas y duraderas que sus padres (tierra, vivienda y animales), pero sujetos a una variada oferta de consumo de bienes móviles (celular, ropa, música y, en casos en los que están más permanentemente habitando la ciudad, por diverso tipo de drogas ilícitas), se debaten en múltiples situaciones caracterizadas por contradicciones y ambigüedades construidas por la intervención estatal a través de una oferta educativa generalmente de baja calidad, por las representaciones mentales comunales mayoritarias y dominantes respecto de la importancia de la instrucción escolar formal pero, sobre todo, por los efectos seductores que ejerce el mercado en su vida cotidiana, principalmente a través de los medios y de lo que ellos experimentan en su relación con sus pares más urbanizados.

Tal condición de indefensión y sobreexposición ante dispositivos estatales, privados y comunales que ven en la posibilidad de educación de las generaciones jóvenes la única manera de salir de la pobreza, y frente a los dispositivos del mercado que los sitúan como uno más de los segmentos de consumidores, produce una inédita condición de socialización juvenil indígena en la que se expresa simultáneamente una descomunalización mercantilizada y una débil integración a un sistema educativo escolarizado precario. Éstas son dos de las dimensiones cruciales que relevan del conjunto de procedimientos etnográficos a través de los que nos hemos relacionado con los adultos, niños y jóvenes de las comunidades indígenas andinas con las que hemos trabajado en lo que corre de la investigación.

QUITO (ECUADOR) — BOGOTÁ (COLOMBIA), FEBRERO 2011

RECIBIDO: FEBRERO 2011

ACEPTADO: MARZO 2011 


\section{REFERENCIAS BIBLIOGRÁFICAS}

AcOSTA, A. (1987): La lógica del capital financiero internacional. Quito: CNE.

AlbORNOZ, O. (1971): Las luchas indígenas en el Ecuador. Guayaquil: Editorial Claridad.

BECK, U. (2002): La sociedad del riesgo global. Barcelona: Paidós.

BourdieU, P. (1996): Cuestiones de sociología. Madrid: Istmo.

FouCAUlt, M. (1986): Historia de la sexualidad I. La voluntad de saber. Barcelona: Paidós.

MARTíneZ, L. (1980): La descomposición del campesinado de la sierra ecuatoriana. Quito: Editorial El Conejo.

- (1984): De campesinos a proletarios. Cambios en la mano de obra rural en la sierra central del Ecuador. Quito: Editorial El Conejo.

- (1992): «Sobre el concepto de comunidad». Cuadernos de la Realidad Ecuatoriana $\mathrm{N}^{\circ}$ 5. Quito.

OBEREM, U. (1979): «Indios libres e indios sujetos a haciendas en la sierra central ecuatoriana a fines de la colonia». Amerikanische Studien I, Haus Volker und Kulturen. St. Augustin.

PlAZA, O. (1981): Formas de dominio, economía y comunidades. Lima: DESCO.

SAlOMON, F. (1981): "Weavers of Otavalo». N. Whitten, Transformations and Ethnicity in Modern Ecuador. Chicago: University of Illinois Press.

SÁNCHEZ PAGA, J. (2007): El movimiento indígena ecuatoriano. Quito: CAAP.

- (2002): Crisis en torno al Quilotoa. Mujer, cultura y comunidad. Quito: CAAP.

— (1993): Transformaciones socioculturales y educación indígena. Quito: CAAP.

(1989): Faccionalismo, organización y proyecto étnico en Los Andes. Quito: CAAP.

- (1984): La trama del poder en la comunidad andina. Quito: CAAP.

SASSEN, S. (2007): Una sociología de la globalización. Madrid: Katz Eds.

UNDA, R. (2010): Jóvenes y juventudes. Acción, representaciones y expectativas sociales de jóvenes en Quito. Quito: Abya Yala.

WODAK, R. (2005): Metodología de análisis crítico del discurso. Madrid: Cátedra. 